\title{
Correction to: Modeling and Simulation with Compose and Activate
}

\section{Correction to:}

\section{S. L. Campbell and R. Nikoukhah, Modeling}

and Simulation with Compose and Activate, https://doi.org/10.1007/978-3-030-04885-3

In the original version of the book, the following corrections have been incorporated:

Additional materials have been included in extras.springer.com and in the copyright page. The extras logo has been included in the cover. The correction book has been updated with the changes. 